\title{
Atuação do terapeuta ocupacional no contexto da hospitalização infantil oncológica: uma revisão da literatura
}

\author{
Operation of occupational therapist in the context of oncological childhood \\ hospitalization: a literature review
}

Actuación de los terapeutas ocupacionales en el contexto de la hospitalización oncológica infantil: una revisión de la literatura

Paula Rayane Oliveira Batista ${ }^{1}$, Raíra Rodrigues da Costa ${ }^{1}$, Tamara Iglesias de Barros ${ }^{1}$, Luzimara Vieira Rodrigues' ${ }^{1}$, Paulo Henrique Barbosa Furtado', Nathalia Castilhos Mello', Sathya da Silva Lobato $^{1}$, Karina Saunders Montenegro ${ }^{1 *}$.

\section{RESUMO}

Objetivo: Identificar como o terapeuta ocupacional pode intervir para minimizar os efeitos da hospitalização infantil oncológica. Métodos: Trata-se de uma revisão bibliográfica integrativa, do tipo descritiva exploratória. O artigo foi constituído por 7 artigos, publicados no período de 2003 a 2019, em língua portuguesa, no qual foram coletados em bases de dados. Como critérios de inclusão, foram usados artigos científicos que apresentassem a temática terapia ocupacional, oncologia infantil ou oncologia pediátrica. Resultados: Dentre os trabalhos reunidos, constatou-se que no ano de 2013 houve um aumento na incidência no porcentual de publicações, com $28,5 \%$, sendo na área do sudeste a ocorrência maior de estudos sobre o tema. Os dados obtidos nesses estudos discutem sobre a importância da intervenção do terapeuta ocupacional, devido este profissional promover um ambiente acolhedor, em meio as rupturas vivenciadas neste período, através principalmente do brincar. Considerações finais: $O$ terapeuta ocupacional é de suma importância para o tratamento da criança com câncer, oferecendo formas na melhoria da qualidade de vida no período de adoecimento e hospitalização.

Palavras-chaves: Terapia Ocupacional, Hospitalização, Infantil.

\begin{abstract}
Objective: Identify how the occupational therapist can intervene to minimize the effects of child oncology hospitalization. Methods: This is a integrative bibliographic review, of an exploratory descriptive. The article consisted of 7 articles, published from 2003 to 2019, in Portuguese, collected in databases. As inclusion criteria, scientific articles using occupational therapeutic therapy, child oncology or pediatric oncology were used. Results: Among the works gathered, completed in 2013, there was an increase in the half-yearly incidence of publications, with $28.5 \%$, with the southeast area having the highest incidence of studies on the topic. The data obtained in the studies discussed on the importance of occupational therapeutic intervention, due to the fact that this professional promotes a welcoming environment, amid the ruptures experienced in this period, mainly through of playing. Final considerations: the occupational therapist is of paramount importance for the treatment of children with cancer, offering ways to improve the quality of life during the period of illness and hospitalization.
\end{abstract}

Keywords: Occupational Therapist, Hospitalization, Infant.

1 Universidade do Estado do Pará, Belém - Pará. *E-mail: karinasmonte@yahoo.com.br

SUBMETIDO EM: 4/2021

ACEITO EM: 5/2021

PUBLICADO EM: 5/2021 


\section{RESUMEN}

Objetivo: Identificar cómo puede intervenir el terapeuta ocupacional para minimizar los efectos de la hospitalización oncológica infantil. Métodos: Se trata de una revisión bibliográfica integrativa, del tipo descriptivo exploratorio. El artículo constaba de 7 artículos, publicados entre 2003 y 2019, en portugués, en los que se recopilaron en bases de datos. Como criterios de inclusión, se utilizaron artículos científicos que presentaron el tema terapia ocupacional, oncología infantil u oncología pediátrica. Resultados: Entre los trabajos reunidos, se encontró que en 2013 hubo un aumento en la incidencia en el porcentaje de publicaciones, con $28.5 \%$, con la mayor ocurrencia de estudios sobre el tema en el área sureste. Los datos obtenidos en estos estudios discuten la importancia de la intervención del terapeuta ocupacional, ya que este profesional promueve un ambiente acogedor, correo electrónico a los descansos experimentados en este período, principalmente a través del juego. Consideraciones finales: El terapeuta ocupacional es extremadamente importante para el tratamiento de niños con cáncer, ya que ofrece formas de mejorar la calidad de vida durante el período de enfermedad y hospitalización.

Palabras clave: Terapeuta Ocupacional, Hospitalización, Infantil.

\section{INTRODUÇÃO}

Segundo Nascimento LC, et al. (2005), o câncer infantil é caracterizado pela proliferação rápida e descontrolada de células anormais. Este processo pode acontecer em qualquer parte do corpo, sendo mais recorrente no sistema sanguíneo, no sistema nervoso central e no sistema linfático. Os fatores de risco estão ligados ao estilo de vida (INSTITUTO NACIONAL DO CÂNCER- INCA, 2018).

No Brasil, estima-se 12.500 novos casos de câncer infanto-juvenil, e está em primeiro lugar dentre os motivos de morte na infância no país. Entretanto, estima-se que em torno de $80 \%$ das crianças e adolescentes acometidos pela doença podem curassem se diagnosticados precocemente (INCA, 2018).

Para Lima MS e Almohalha L (2011) é imprescindível destacar que o processo de hospitalização é uma importante etapa do tratamento. Porém, potencialmente traumático, a criança estará sujeita a situações de difícil controle, vivenciando de diferentes sentimentos - em sua maioria negativos - frente a procedimentos terapêuticos desconhecidos e invasivos, que podem causar sintomas de anormalidade fisiológica e sofrimento psíquico, os quais podem tornar-se patológicos proporcionando prejuízos na qualidade de vida, no bem-estar e também comprometem a autonomia e a independência da criança (MOTTA AB e ENUMO SRF, 2004; OLIVEIRA ACSS e CAVALCANTE MCV, 2015; SILVA AB, et al., 2021; NORENA AL e SIBANAL L, 2011; DIAS JJ, et al., 2013).

Conforme Lima MS e Almohalha L (2011), o terapeuta ocupacional deve intervir para que a criança continue se desenvolvendo, ainda que a doença lhe cause restrições, dando ênfase aos recursos terapêuticos que proporcionam o brincar. Considerando-se que o brincar é a ocupação primordial da criança e faz parte do desenvolvimento infantil, além de diminuir o estresse, o medo e a ansiedade decorrentes do ambiente hospitalar (GARCIA-SCHINZARI NR, et al., 2013; LIMA MS e ALMOHALHA L, 2011 apud VASCONCELOS et al., 2006).

Assim, o presente trabalho visa identificar a atuação do terapeuta ocupacional na hospitalização infantil oncológica. E pretende-se responder a seguinte pergunta norteadora: Como o terapeuta ocupacional pode intervir para minimizar os efeitos da hospitalização infantil oncológica?

\section{MÉTODOS}

O artigo é uma revisão bibliográfica integrativa do tipo descritiva exploratória. O levantamento dos dados foi feito no site oficial do Instituto Nacional do Câncer (INCA) e Organização Mundial da Saúde (OMS), nas bases de dados do Literatura Latino-americana e do Caribe em Ciências da Saúde (LILACS) e Livraria Cientifica Eletrônica Online (SciELO), além do uso dos periódicos Caderno de Terapia Ocupacional da 
Universidade Federal de São Carlos (UFSCar) e Revista de Terapia Ocupacional da Universidade de São Paulo (USP).

Utilizou-se os descritores: Terapia Ocupacional, Oncologia Infantil e Oncologia Pediátrica. Foram critérios de inclusão: artigos científicos que abordassem a temática Terapia Ocupacional, oncologia infantil ou oncologia pediátrica, tais termos deveriam aparecer no título, resumo e/ou palavras chaves, publicados no período de 2003 a 20019, escritos em língua portuguesa, artigos brasileiros. Foram critérios de exclusão artigos que não estão nesse período, artigos em língua estrangeira, artigos incompletos, artigos pagos, carta ao editor, descrição de congresso, simpósio e palestra.

A análise dos dados quantitativos foi registrada em frequência e porcentagem correspondendo ao: ano de publicação, instituição, local de publicação e tipos de estudo. Esses dados foram apresentados em tabelas que ajudaram na identificação do perfil dos trabalhos produzidos com essa temática nos últimos 16 anos.

A análise dos dados qualitativos foi feita a partir da leitura detalhada dos artigos na tentativa de responder à pergunta norteadora do presente estudo. Os dados foram organizados em três categorias de análise: 0 brincar no ambiente infantil oncológico hospitalar, humanização do tratamento de oncologia infantil no contexto hospitalar e desenvolvimento infantil, terapia ocupacional e oncologia pediátrica.

\section{RESULTADOS E DISCUSSÃO}

Durante a análise dos dados foram encontrados o total de 12.441 artigos, porém, apenas 24 atenderam os critérios de inclusão e exclusão. Entretanto após a leitura dos mesmos observou- se que havia artigos repetidos, desse modo, sendo estes excluídos restaram sete artigos (Tabela 1).

Tabela 1 - Descritores após critérios de inclusão e exclusão nas bases de dados.

\begin{tabular}{lccccc}
\hline & Lilacs & OMS & Scielo & UFSCar & USP \\
\hline Oncologia Infantil & 0 & 0 & 0 & 1 & 0 \\
\hline Oncologia Pediátrica & 0 & 0 & 0 & 1 & 4 \\
\hline Terapia Ocupacional & 2 & 1 & 0 & 2 & 5 \\
\hline Terapia Ocupacional e Oncologia Infantil & 0 & 0 & 0 & 1 & 0 \\
\hline Terapia Ocupacional e Oncologia Pediátrica & 1 & 1 & 0 & 1 & 4 \\
\hline
\end{tabular}

Legenda: OMS, Organização Mundial da Saúde.

Fonte: Batista PRO, et al., 2021.

Através de pesquisa identificou-se que a maioria dos trabalhos selecionados ocorreu no ano de 2013, havendo um aumento de 28,5\% comparado aos anos de 2009, 2011, 2012, 2015 e 2017 que tiveram apenas $14,2 \%$ de artigos publicados, apontando um crescimento de pesquisas no ano de 2013 e logo após uma diminuição nos anos seguintes (Tabela 2).

Tabela 2 - Ano de Publicação.

\begin{tabular}{ccc}
\hline Ano & Ocorrência & Percentual \\
\hline $\mathbf{2 0 0 9}$ & 1 & $14,2 \%$ \\
\hline $\mathbf{2 0 1 1}$ & 1 & $14,2 \%$ \\
\hline $\mathbf{2 0 1 2}$ & 1 & $14,2 \%$ \\
\hline $\mathbf{2 0 1 3}$ & 2 & $28,5 \%$ \\
\hline $\mathbf{2 0 1 5}$ & 1 & $14,2 \%$ \\
\hline $\mathbf{2 0 1 7}$ & 1 & $14,2 \%$ \\
\hline
\end{tabular}

Fonte: Batista PRO, et al., 2021. 
De acordo com os dados do INCA (2016) somente no Brasil ocorreram, em 2014, aproximadamente, 2.724 óbitos por câncer em crianças e adolescentes, de 0 a 19 anos, e consequentemente o aumento dos cuidados destinados a esse público. Assim é preocupante que a produção de pesquisas na área não acompanhe a adição de casos da doença, ocasionando dessa forma poucos trabalhos divulgados.

É por meio de pesquisas que ocorrerá a identificação e análise das dificuldades principais em uma área determinada. Ademais, faz-se notório a importância de investigações para apontar as modificações básicas do cotidiano dessas crianças, como seu desempenho ocupacional será afetado e a adequação da mesma perante essa nova realidade, assim como o aperfeiçoamento das formas de trabalho voltadas ao público infantil oncológico.

Tabela 3 - Instituição de publicação.

\begin{tabular}{ccc}
\hline Instituição & Ocorrência & Percentual \\
\hline USP & 4 & $57,1 \%$ \\
\hline UFSCar & 2 & $28,5 \%$ \\
\hline UFTM & 1 & $14,2 \%$ \\
\hline
\end{tabular}

Fonte: Batista PRO, et al., 2021.

Considerando a produção de artigos publicados por local de publicação, constatou-se que todos os artigos selecionados para estudo foram publicados na região Sudeste, com o quantitativo de $57,01 \%$ na Universidade de São Paulo, 28,5\% na Universidade Federal de São Carlos e 14,2\% na Universidade Federal do Triângulo Mineiro (Tabela 3).

Tabela 4 - Local de Publicação.

\begin{tabular}{lll}
\hline Unidade federativa & Ocorrência & Percentual \\
\hline São Paulo & 6 & $85,7 \%$ \\
\hline Minas Gerais & 1 & $14,3 \%$
\end{tabular}

Fonte: Batista PRO, et al., 2021.

Considerando a produção de artigos publicados por local de publicação, constatou-se que os artigos selecionados para o estudo foram publicados na região Sudeste, com o quantitativo de $85,7 \%$ no estado de São Paulo e 14,3\% no estado de Minas Gerais (Tabela 4).

Observa-se o aumento de estudos e publicações na região Sudeste, tal dado é significativo se for levado em consideração a amplitude do Brasil e sua variação a nível regional, cultural e social que fundamentam a realização de estudos na área. Em vista disso, vale pontuar a necessidade do desenvolvimento de estudos nas outras regiões voltadas para a capacitação dos profissionais de acordo com as necessidades de cada criança.

No que se refere ao tipo de estudo $42,85 \%$ são de pesquisas do tipo Descritiva quanti qualitativa; $14,28 \%$ Pesquisa-ação; $14,28 \%$ pesquisa de campo do tipo horizontal; $14,28 \%$ pesquisa de campo qualitativa descritiva e $14,28 \%$ clínico qualitativa.

Portanto, os tipos de pesquisa realizadas são de suma importância para a apuração dos fatos no que diz respeito a temática abordada, assim como, a constatação das dificuldades enfrentadas pelo público investigado, objetivando a explicação do problema pesquisado.

Analisando os dados apresentados e discutidos nos estudos identificou-se que os artigos A1, A2, A3 e A7 obtiveram resultados similares tendo como referência o brincar. Todos destacaram-no como uma ocupação de suma importância na infância. Destaca-se que os artigos A1 e A2 apresentaram mais concordâncias nos resultados quanto a atuação do Terapeuta Ocupacional no ambiente hospitalar com o público infantil (GARCIA 
NR, et al., 2012; GIARDINETTO ARSB, et al., 2009; JOAQUIM RHVT, et al., 2017; LIMA MS e ALMOHALHA L, 2011).

Os artigos A6 e A7 apresentaram similaridades quanto aos seus objetos de estudo, onde o primeiro objetivou a apresentação da avaliação vivenciada pelos participantes e pesquisadores em uma comunidade durante um período de tempo. E o segundo utilizou-se de um questionário semiestruturado e auto aplicado, via internet para a obtenção de dados (GALHEIGO SM, et al., 2015; JOAQUIM RHVT, et al., 2017).

Os artigos A1, A2, A7 priorizaram demonstrar a atuação do terapeuta ocupacional, enquanto o A3 objetivou identificar a visão dos profissionais da saúde de uma enfermaria oncopediátrica em relação ao "Programa de extensão Caixa de histórias" no cuidado à criança hospitalizada, programa este desenvolvido por terapeutas ocupacionais (GARCIA NR, et al., 2012; GIARDINETTO ARSB, et al., 2009; JOAQUIM RHVT, et al., 2017; LIMA MS e ALMOHALHA L, 2011).

O artigo A4 apresentou destaque frente aos demais por enfatizar a experiência humana e a construção de significados partindo disto, enquanto o A5 visou destacar a identificação, inserção e ampliação da atuação terapêutica ocupacional no Estado de São Paulo voltada para a área oncológica no contexto hospitalar, tendo como base as Políticas Públicas de Atenção Oncológica (MOTTA MR, et al., 2013; SILVA LF e GIARDINETTO ARSB, 2013).

Após a análise dos artigos encontrados conclui-se que, a maioria foi publicada no ano de 2013 (28,5\%), com uma incidência maior de estudos sobre o tema na região sudeste, no Estado de São Paulo e Minas gerais, nas Universidade de São Paulo, Universidade Federal de São Carlos e Universidade Federal do Triângulo Mineiro. Além disso, quanto ao tipo de estudo predominante, foi a pesquisa descritiva quanti qualitativa, com $42,85 \%$.

Com o intuito de responder à pergunta norteadora dessa pesquisa, foi realizada a leitura exaustiva dos sete artigos selecionados sendo possível comparar os achados com a literatura da área. Assim, foi possível elaborar uma resposta para a pergunta norteadora: como o terapeuta ocupacional pode intervir para minimizar os efeitos da hospitalização infantil oncológica?

De acordo com o artigo A1, desde que chegou ao Brasil, a Terapia Ocupacional tem expandido sua atuação a diversos campos, sendo um deles o campo da hospitalização infantil oncológica. Nesse contexto, a importante atuação do terapeuta ocupacional consiste em promover saúde e qualidade de vida para a criança que está hospitalizada, além de contribuir para que o desenvolvimento desse paciente não sofra grandes prejuízos decorrentes da mudança abrupta do processo de tratamento oncológico (GIARDINETTO ARSB, et al., 2009).

Uma das práticas realizadas e incentivadas pelo terapeuta é o brincar. Esta prática é considerada como sendo de suma importância, visto que, é a principal ocupação da infância, de acordo com os artigos A1, A2, A3 e A7.

O brincar também é válido para possibilitar e fortalecer a relação entre o terapeuta e a criança, o que facilita a aplicação de outras abordagens terapêuticas que poderão ser necessárias e aumenta a chance de sucesso no processo terapêutico, visto que, dessa forma haverá maiores chances de ocorrer um atendimento mais holístico e humanizado, conforme o artigo A1 (GIARDINETTO ARSB, et al., 2009).

Segundo o artigo A2, durante a intervenção do terapeuta ocupacional em hospitalização oncológica, é necessário trabalhar a independência e a autonomia da criança. Também destacam a importância de se oportunizar momentos prazerosos dentro do ambiente hospitalar para que a criança e seus cuidadores se sintam acolhidos nesse ambiente, que muitas vezes é visto como agressivo (LIMA MS e ALMOHALHA L, 2011).

Ainda acerca da humanização, é cabível ao terapeuta ocupacional, não só agir no contexto, mas também na estrutura física do local para torná-lo mais tolerável. É importante que o ambiente seja agradável. O bemestar da criança no ambiente hospitalar pode ser potencializado por desenhos, de autoria da criança, fixados nas paredes, ou até mesmo por brinquedos que o infante tenha apreço. O chamado tratamento paliativo é 
igualmente conveniente e reforça a humanização no contexto de hospitalização infantil oncológica. Portanto, seguindo a argumentação do artigo A2, conclui-se que a prevenção e o alívio do sofrimento trazido pela doença também são meios que o terapeuta ocupacional utiliza (LIMA MS e ALMOHALHA L, 2011).

Visto que, de acordo com Garcia NR, et al. (2012), o terapeuta ocupacional é capacitado para lidar e contribuir em todas as fases do desenvolvimento infantil, pode-se afirmar que através da promoção do bemestar e do aumento da autoestima do paciente, o terapeuta ocupacional auxilia de forma significativa na qualidade do tratamento infantil oncológico e na minimização dos efeitos desse processo.

A análise realizada a partir desta pesquisa mostra que o agravo causado pelo tratamento invasivo acarreta consequências negativas. Sendo assim, o terapeuta ocupacional age para possibilitar um ambiente mais ameno tanto para a criança quanto para seus familiares, fazendo com que o cliente seja mais "flexível" e contribua com o tratamento. Um ambiente hospitalar humanizada ajuda a tornar viável uma relação agradável e útil entre paciente-terapeuta-família.

Diante do que foi exposto, enfatiza-se que o terapeuta ocupacional atua através de diversas formas de intervenção e reabilitação no campo oncológico, e que essa atuação ocorre principalmente por meio do brincar. Dessa maneira, destaca-se a importância das atividades lúdicas com crianças hospitalizadas como um fator de grande relevância no desenvolvimento físico, cognitivo, psicológico e sensório-motor desses pacientes, além de influenciar na humanização nesse contexto.

Apenas os artigos A1, A2, A4, A7 abordaram a temática da pergunta norteadora, o que se considera um quantitativo reduzido. Considerando que o tema tem imensa relevância, é perceptível que existe a necessidade da promoção de discussões e pesquisas voltadas para o trabalho do terapeuta ocupacional com o público infantil oncológico no contexto da hospitalização.

Para a criação das categorias de análise, foram utilizados sete artigos que abordassem a temática terapia ocupacional, oncologia infantil ou oncologia pediátrica, buscando uma melhor compreensão sobre o tema abordado.

\section{O brincar no ambiente infantil oncológico hospitalar}

Segundo a Lei no 11.104, de 21 de março de 2005 (BRASIL, 2005) é obrigatório a instalação de uma brinquedoteca em ambientes da saúde que ofereçam internação pediátrica. Sendo assim, para os artigos A1, A2, A3 e A7 o brincar para uma criança é importante por ser uma das principais atividades do infante, sendo muito relevante trazer essa atividade para o público infantil em tratamento oncológico hospitalizadas, visto que estão limitados a esse ambiente e o brincar funciona como um facilitador na transformação do cotidiano da hospitalização (GARCIA-SCHINZARI, et al., 2013; GARCIA NR, et al., 2012).

Além disso, o brincar é utilizado para manter uma melhor relação entre o terapeuta ocupacional, outros profissionais e o paciente, tendo em vista que a maioria dos procedimentos que esse público afetado faz são invasivos, com agulhas, cateteres, dores. Sendo assim, como referido no artigo A1, os profissionais que entendem a importância das atividades lúdicas para as crianças hospitalizadas, estabelecem uma relação mais harmônica com a criança, sabendo tratar o paciente de uma maneira mais humanizada, proporcionando assim qualidade de vida ocupacional (GARCIA-SCHINZARI, et al., 2013; GIARDINETTO ARSB, et al., 2009).

Dessa forma, de acordo com o artigo A2, A3 e A7 é por meio da brinquedoteca, atividades em grupo ou inclusive no leito, dentro dos hospitais, que as crianças podem exercer melhor essa atividade, tudo conforme o quadro clínico da criança e com sua idade (GIARDINETTO ARSB, et al., 2009). Ademais, pode ser um meio de socialização com outros pacientes oncológicos, ajudando na sua capacidade de interação social, pois a criança está limitada dos seus núcleos sociais, como familiares e escolares, com uma ruptura do seu cotidiano diário (JOAQUIM RHVT, et al., 2017). Portanto, esse ambiente ajuda a criança em seu ânimo prosperando o seu estado emocional, e com mais vontade de curar- se (GARCIA NR, et al., 2012). Em suma, esse ambiente pode servir para o melhor acolhimento do infante, utilizando estratégicas lúdicas, além de servir para a diminuição dos impactos negativos da internação e estimula o desenvolvimento infantil (NUNES CJRR, et al., 2013). 


\section{Humanização do tratamento de oncologia infantil no contexto hospitalar}

Para Giardinetto ARSB, et al. (2009) o terapeuta ocupacional com sua intervenção holística tem o papel de amenizar os agravos decorrentes do processo de tratamento da criança com câncer. $\mathrm{O}$ artigo A2 discorre a respeito do ambiente e de como o terapeuta ocupacional pode torná-lo mais agradável para o seu cliente através da decoração do mesmo, fazendo com que a criança se sinta mais à vontade e menos rígida ao tratamento. $\mathrm{O}$ artigo $\mathrm{A} 1$ confirma através do relato de outros profissionais que após a participação do terapeuta ocupacional na oncologia pediátrica, as crianças ficaram mais alegres e adeptas ao seu tratamento.

Motta MR, et al. (2013) destacam que o terapeuta ocupacional busca estimular é a subjetividade e criatividade da criança, para que não atrapalhe o seu desenvolvimento. $\mathrm{O}$ artigo $\mathrm{A} 4$ retrata que através de atividades e do próprio brincar da criança, o terapeuta ocupacional pode estimular esses aspectos que são de grande importância para que a criança se sinta acolhida e mais acessível no ambiente hospitalar.

O artigo A2 exemplifica que se a família não estiver lidando adequadamente com o processo de tratamento, a reabilitação da criança pode ficar comprometida. Diante disso, faz-se necessário que os indivíduos envolvidos no processo de adoecimento sejam alvo da intervenção profissional, para que recebam 0 apoio adequado e possam oferecer o cuidado para a criança, sem que seu sofrimento perpasse para a mesma (LIMA MS e ALMOHALHA L, 2011).

Após a análise dos artigos conclui-se que o terapeuta ocupacional é necessário para realizar o acolhimento humanizado na oncologia pediátrica, podendo se utilizar de inúmeros recursos visando sempre estimular o desenvolvimento e o cuidado adequado para cada indivíduo (LIMA MS e ALMOHALHA L, 2011).

\section{Desenvolvimento infantil, Terapia Ocupacional e Oncologia Pediátrica}

Segundo Joaquim RHVT, et al. (2017) no A7, a infância é um período de extrema importância para o desenvolvimento do ser humano em relação a aspectos físicos, motores, sociais, psicológicos e emocionais, e o crescimento humano depende da maturação biológica associada com a estimulação oferecida pelo meio ambiente desde os primeiros anos de vida.

De acordo com Lima MS e Almohalha L (2011) apud Vasconcelos, et al., (2006) quando uma criança é hospitalizada por um longo período, nesse caso, devido ao quadro oncológico, ela pode apresentar rupturas ou perdas definitivas das habilidades da vida diária, perda de interesse e vontade, uma vez que abruptamente ela é afastada da família, dos amigos e da escola. Visto que, o tratamento oncológico requer uma constância e demanda tempo para ser concluído, surge, a partir disso, dificuldade para a ida dos infantes e juvenis à escola formal (MENDES MVC, et al., 2018).

Para Silva IF e CABRAL IE (2014) suas referências afetivas e sociais são perdidas e ela passa a enfrentar uma realidade na qual é submetida a exames, internações hospitalares prolongadas e tratamentos dolorosos invasivos, como a quimioterapia, radioterapia e cirurgias. Em virtude disso, o terapeuta ocupacional adentra em sua área de atuação, visto que é capacitado para analisar e avaliar o desenvolvimento infantil e tem papel fundamental em intervenções com crianças no contexto hospitalar. Uma de suas ações terapêuticas é o uso de atividades lúdicas e criativas, pois são recursos que promovem a continuidade do desenvolvimento global, da saúde mental da criança hospitalizada, a reorganização do cotidiano, a expressão da dor, da tristeza ou da desolação e favorece vínculo entre paciente-terapeuta-família (JOAQUIM RHVT, et al., 2017).

Portanto, como afirma o A2, a promoção do desenvolvimento infantil deve ocorrer durante todo 0 tratamento, com o intuito de amenizar os impactos negativos já existentes no cotidiano de uma criança hospitalizada e garantir que a mesma desempenhe seus papéis ocupacionais em cada fase, além de exercer o seu direito de viver com qualidade.

Contudo, a hospitalização é um dos mais importantes processos do tratamento oncológico, a qual rompe com o cotidiano trazendo, na infância, alterações árduas nos diversos componentes do desempenho ocupacional, cognitivos, biopsicossociais e sensório-motores - gerando, portanto, impacto negativo não somente no paciente, como também na família. Nesse sentido, o terapeuta ocupacional avalia e intervém, de 
maneira holística e humanizada, esses componentes através principalmente do brincar, sendo a ocupação primordial da criança.

\section{CONSIDERAÇÕES FINAIS}

Atuação do terapeuta ocupacional é de suma importância para o desenvolvimento da criança hospitalizada e com diagnóstico de câncer, pois a hospitalização Ihe provocará uma quebra em seus aspectos físicos, sociais, psicológicos, motores e emocionais porque é na infância que há a maior atividade neurológica associada a estimulação para que ocorra o desenvolvimento. No entanto, devido a hospitalização haverá rupturas ou perdas quanto a esse desenvolvimento neuropsicomotor. Assim, o terapeuta ocupacional se fará necessário atuando através das atividades lúdicas proporcionando estimulação e promovendo o desenvolvimento do cliente.

\section{REFERÊNCIAS}

1. BRASIL, Ministério da Saúde. Lei 11.104/2005, de 2005. Disponível em: http://bvsms.saude.gov.br/bvs/saudelegis/gm/2005/prt2261_23_11_2005.html. Acessado em: 07 de abril 2021.

2. DIAS JJ, et al. A experiência de crianças com câncer no processo de hospitalização e no brincar. Revista Mineira de Enfermagem, 2013; 17(3): 608-613.

3. GALHEIGO SM, et al. Comunidade de prática de Terapia Ocupacional: a avaliação do processo pelos participantes e pelos pesquisados. Cad. Ter. Ocup. UFSCar, São Carlos, 2015; 23(3): 463-474.

4. GARCIA-SCHINZARI NR, et al. Cuidados Paliativos junto a Crianças e Adolescentes Hospitalizados com Câncer: 0 Papel da Terapia Ocupacional. Revista Brasileira de Cancerologia, 2013; 59(2): 239-247.

5. GARCIA NR, et al. As caixas de histórias na visão de profissionais de saúde como estratégia de enfrentamento da hospitalização infantil. Revista De Terapia Ocupacional Da Universidade De São Paulo, 2012; 23(2): 169-177.

6. GIARDINETTO ARSB, et al. A importância da atuação da Terapia Ocupacional com a população infantil hospitalizada. UFSCar, 2009; 17(1).

7. INSTITUTO NACIONAL DO CÂNCER. Incidência, mortalidade e morbidade hospitalar por câncer em crianças, adolescentes e adultos jovens no Brasil; informações dos registros de câncer e do sistema de mortalidade, 2016.

8. INSTITUTO NACIONAL DO CÂNCER. Tipos de câncer: Câncer infanto-juvenil, 2018.

9. JOAQUIM RHVT, et al. Terapia Ocupacional e Oncologia pediátrica: Caracterização dos profissionais em centros de referência no Estado de São Paulo. Revista de Terapia Ocupacional da Universidade de São Paulo, 2017; 28 (21).

10. LIMA MS, ALMOHALHA L. Desvelando o papel do terapeuta ocupacional na oncologia pediátrica em contextos hospitalares. Revista De Terapia Ocupacional Da Universidade De São Paulo, 2011; 22(2): 172-181.

11. MENDES MVC, et al. Crianças e Adolescentes em Tratamento Oncológico: uma Análise sobre a Visão do Adiamento do Início ou Interrupção da Educação Escolar. Revista Brasileira de Cancerologia, 2018; 64(3): 301-309.

12. MOTTA, AB, ENUMO, SRF. Câncer infantil: uma proposta de avaliação as estratégias de enfrentamento da hospitalização. Campinas, 2004; 21(3): 193-202.

13. MOTTA MR, et al. O processo criativo de pacientes internados para tratamento quimioterápico: uma contribuição a partir do pensamento de D.W. Winnicott. Revista de Terapia Ocupacional da USP, 2013; 24(2).

14. NASCIMENTO LC, et al. Crianças com câncer e suas famílias. Rev. da Escola de Enfermagem da Universidade de São Paulo, 2005; 39(4).

15. NORENA AL, CIBANAL L. A experiência de crianças hospitalizadas sobre sua interação com os profissionais de enfermagem. Revista Latino-Americana de Enfermagem, 2011; 19(6).

16. NUNES CJRR, et al. A importância da brinquedoteca hospitalar e da Terapia Ocupacional sob a óptica da equipe de enfermagem de um hospital público do Distrito Federal. Cad. Ter. Ocup, UFSCar, 2013; $21: 3$.

17. OLIVEIRA ACSS, CAVALCANTE MCV. Intervenção da Terapia Ocupacional junto à criança hospitalizada. Revista de Pesquisa em Saúde, 2015; 16(1).

18. SILVA AB, et al. Repercussões emocionais em pacientes em seguimento oncológico: ansiedade, depressão e qualidade de vida. Revista Eletrônica Acervo Saúde, 2021; 13(3).

19. SILVA ACC, GIARDINETTO, ARSB. Políticas públicas em oncologia: refletindo sobre a atuação da terapia ocupacional. Revista De Terapia Ocupacional Da Universidade De São Paulo, 2013;23 (3).

20. SILVA IF, CABRAL IE As repercussões do câncer sobre o brincar da criança: implicações para o cuidado de enfermagem. Texto contexto enfermagem, 2014; 23(4): 935-43. 